\title{
Shorter regimens for multidrug-resistant tuberculosis should also be applicable in Europe
}

\author{
To the Editor:
}

Since May 2016, the World Health Organization (WHO) has recommended shorter regimens to treat rifampicin-resistant/multidrug-resistant tuberculosis (RR/MDR-TB), substantially reducing the treatment duration to 9-12 months [1]. VAN DER WERF et al. [2] estimated that only $11 \%$ of RR/MDR-TB patients in the European Union (EU)/European Economic Area (EEA) fulfilled the WHO inclusion criteria, which is similar to estimates by LANGE et al. [3] and Sotgiu et al. [4]. This estimate raises concerns, as that the conventional long-duration regimen has very poor results in the EU/EEA. Health sector crisis, increasing inequality and xenophobia add to the urgency to prevent and manage MDR-TB. The main exclusion criteria are as follows.

1) Previous use of second-line drugs (SLDs). The estimate is clearly too high (55\% of RR/MDR-TB cases), as it also excluded previous use of only first-line drugs. A better estimate could be from countries like Latvia where $42 \%$ of RR/MDR-TB cases in 2005-2015 were previously treated, $30 \%$ of them with SLDs, representing $12 \%$ of all RR/MDR-TB cases (V. Riekstina, Centre of Tuberculosis and Lung Diseases, Riga, Latvia; personal communication). In Western Europe, many patients with RR/MDR-TB were born in Asia and Africa, where SLDs have been used far less frequently [5].

2) Resistance to second-line injectable drugs (SLIDs) and/or fluoroquinolones. Such resistance affects $41 \%$ of RR/MDR-TB patients in the EU/EEA; $34 \%$ in new patients and $48 \%$ in previously treated patients (communication with the European Centre for Disease Prevention and Control (ECDC), Stockholm, Sweden) (table 1). In patients of Former Soviet Union (FSU) origin, resistance levels are higher $(50 \%$ in all, $44 \%$ in new and $56 \%$ in previously treated patients). In patients whose origin was outside the FSU (64\% of all cases), the level of resistance was considerably lower (34\% overall, $22 \%$ in new and $42 \%$ in previously treated patients). The proportion in previously treated patients is even lower, if patients treated with SLDs are excluded first (criterion 1). Furthermore, all ofloxacin-resistant cases were also considered to be moxifloxacin-resistant, but only $7 \%$ of ofloxacin-resistant strains have been found to be resistant to moxifloxacin [6]. In a study performed in Bangladesh, only high-level fluoroquinolone resistance reduced success, caused by failure [7]. Most of the mutations correlating with this high-level resistance can be identified by the line-probe assay recommended by the WHO [8].

3) Resistance to other drugs in the regimen, particularly ethambutol or pyrazinamide [1]. In the cohort studies performed in Bangladesh [7] and West Africa [9], on which the recommendation for the shorter regimens is based, cases with resistance to isoniazid, ethambutol or pyrazinamide were not excluded and the overall success was high. Moreover, WHO does not recommend that treatment decisions should be based on drug-susceptibility test (DST) results for ethambutol as the test is unreliable [10]. There is no approved rapid test for pyrazinamide resistance and the clinician may decide to use the shorter MDR-TB regimen in its presence [10]. Susceptibility to other drugs, such as clofazimine, is either never tested routinely, or their DST results are notoriously unreliable and their testing is therefore discouraged. In the EU/EEA, DST coverage is fortunately high (fulfilling WHO criteria for exclusion of resistance to SLID and fluoroquinolones), meaning resistance to ethambutol and pyrazinamide would therefore be a major reason for exclusion.

@ERSpublications

Shorter MDR-TB regimens should not be excluded because of resistance to first-line drugs or extrapulmonary sites http://ow.ly/E4oo30bsIea

Cite this article as: Heldal E, Van Deun A, Chiang C-Y, et al. Shorter regimens for multidrug-resistant tuberculosis should also be applicable in Europe. Eur Respir J 2017; 49: 1700228 [https://doi.org/10.1183/ 13993003.00228-2017]. 
TABLE 1 Tuberculosis (TB) drug resistance according to each patient's treatment history and place of origin in rifampicinresistant/multidrug-resistant (RR/MDR)-TB cases registered in European Union/European Economic Area countries, 2010-2014

\begin{tabular}{|c|c|c|c|c|c|c|c|c|c|c|c|c|}
\hline \multirow[t]{2}{*}{$\begin{array}{r}\text { Place of } \\
\text { origin }\end{array}$} & \multirow[t]{2}{*}{$\begin{array}{l}\text { Treatment } \\
\text { history }\end{array}$} & \multirow[t]{2}{*}{$\begin{array}{l}\text { Notified } \\
\text { cases } \mathrm{n}\end{array}$} & \multicolumn{2}{|c|}{$\mathrm{Km}$ or $\mathrm{Am}$} & \multicolumn{2}{|c|}{ Mfx or Ofx } & \multicolumn{2}{|c|}{$\begin{array}{l}\mathrm{Km} \text { or Am and/ } \\
\text { or Mfx or Ofx }\end{array}$} & \multicolumn{2}{|c|}{ Ethambutol } & \multicolumn{2}{|c|}{$\begin{array}{c}\text { Sensitive to all } \\
\text { drugs }\end{array}$} \\
\hline & & & Tested $\mathbf{n}$ & $\% \mathbf{R}$ & Tested $\mathbf{n}$ & $\% \mathbf{R}$ & Tested $\mathbf{n}$ & $\% \mathbf{R}$ & Tested $\mathbf{n}$ & $\% \mathbf{R}$ & Tested $\mathbf{n}$ & $\% \mathrm{~S}$ \\
\hline & Total & 4859 & 2475 & 25.3 & 1986 & 21.2 & 2491 & 33.6 & 2443 & 59.6 & 1927 & 44.9 \\
\hline & New & 1800 & 940 & 14.4 & 856 & 13.4 & 947 & 21.6 & 937 & 49.3 & 840 & 51.4 \\
\hline & Previously treated & 2866 & 1439 & 33.6 & 1033 & 28.3 & 1446 & 42.3 & 1410 & 67.2 & 994 & 39.0 \\
\hline & New & 1303 & 1128 & 34.0 & 1128 & 20.4 & 1129 & 43.8 & 1127 & 68.8 & 1125 & 22.1 \\
\hline & Previously treated & 1253 & 1070 & 41.5 & 1073 & 39.0 & 1073 & 56.0 & 1073 & 72.1 & 1069 & 16.1 \\
\hline & Unknown history & 135 & 74 & 43.2 & 74 & 32.4 & 75 & 52.0 & 73 & 67.1 & 71 & 22.5 \\
\hline \multicolumn{13}{|l|}{ Total } \\
\hline & Total & 7550 & 4747 & 31.3 & 4261 & 25.7 & 4768 & 41.4 & 4716 & 64.8 & 4192 & 31.1 \\
\hline
\end{tabular}

Km: kanamycin; Am: amikacin; Mfx: moxifloxacin; Ofx: Ofloxacin; R: resistant; S: sensitive; FSU: former Soviet Union. ${ }^{\#}$ : FSU includes Armenia, Azerbaijan, Belarus, Estonia, Georgia, Kazakhstan, Kyrgyzstan, Latvia, Lithuania, Moldova, Russia, Tajikistan, Turkmenistan, Ukraine, Uzbekistan. Source: European Centre for Disease Prevention and Control (ECDC).

4) An extrapulmonary site, with exclusion based on lack of data. The rifampicin-based short-course chemotherapy was first developed for pulmonary TB in the 1970s and 1980s [11], and later studies found that it was also effective in extrapulmonary forms (with the exception of TB meningitis) [12]. There is no apparent reason to postulate that the shorter MDR-TB regimen will not work in extrapulmonary TB.

Shortened MDR-TB regimens have been shown to protect against acquired resistance to SLD (one in 515 cases in Bangladesh) [7]. As they do not include new drugs, they will be protected and provide life-saving treatments for patients with resistance or adverse reactions to SLDs. By the end of 2015, bedaquiline had already been used in 70 countries; failures and resistance development has been reported [13].

Specialists may be reluctant to use standardised regimens [14]. When DOTS (directly observed treatment, short course) was introduced globally in the 1990s, standard regimens had already long been adopted in leading European countries without diminishing the role of specialists in providing holistic treatment for their patients. It could and should be analogous with the standardised shorter MDR-TB regimens. Specialists may treat most of the patients with shorter regimens at low cost, leaving more time and funding for the increasing number of patients with resistance to SLID or fluoroquinolones, as well as adverse reactions.

In Norway, the National MDR-TB Technical Group recently included the shorter regimens in the treatment recommendations (unpublished data). WHO criteria are generally interpreted to exclude most patients because of resistance to ethambutol, pyrazinamide or an extrapulmonary site. This forces the patient to be enrolled on the conventional regimen with twice the duration and documented unacceptable results. The shorter regimens should also be eligible for patients with strains resistant to ethambutol or pyrazinamide (not to contradict the latest WHO recommendation) and should be evaluated in patients with an extrapulmonary site (under operational research conditions) in EU/EEA countries, adding much needed evidence about the regimens from another setting.

Einar Heldal ${ }^{1}$, Armand Van Deun ${ }^{2,3}$, Chen-Yuan Chiang ${ }^{3,4,5}$ and Hans L. Rieder ${ }^{6}$

${ }^{1}$ Norwegian Institute of Public Health, Oslo, Norway. ${ }^{2}$ Mycobacteriology Unit, Institute of Tropical Medicine, Antwerp, Belgium. ${ }^{3}$ International Union Against Tuberculosis and Lung Disease, Paris, France. ${ }^{4}$ Division of Pulmonary Medicine, Dept of Internal Medicine, Wan Fang Hospital, Taipei Medical University, Taipei, Taiwan. ${ }^{5}$ Dept of Internal Medicine, School of Medicine, College of Medicine, Taipei Medical University, Taipei, Taiwan. ${ }^{6}$ Epidemiology, Biostatistics and Prevention Institute, University of Zurich, Zurich, Switzerland.

Correspondence: Einar Heldal, Arvollveien 60D, 0590 Oslo, Norway. E-mail: Einar.heldal@gmail.com

Received: Jan 312017 | Accepted after revision: March 062017

Conflict of interest: None declared. 


\section{References}

1 World Health Organization. WHO treatment guidelines for drug-resistant tuberculosis - 2016 update. Geneva, World Health Organization, 2016.

2 van der Werf MJ, Hollo V, Ködmön C, et al. Eligibility for shorter treatment of multidrug-resistant tuberculosis in the European Union. Eur Respir J 2017; 49: 1601992.

3 Lange C, Duarte R, Fréchet-Jachym M, et al. Limited benefit of the new shorter multidrug-resistant tuberculosis regimen in Europe. Am J Respir Crit Care Med 2016; 194: 1029-1031.

4 Sotgiu G, Tiberi S, D'Ambrosio L, et al. Faster for less: the new "shorter" regimen for multidrug-resistant tuberculosis. Eur Respir J 2016; 48: 1503-1507.

5 Jensenius M, Winje BA, Blomberg B, et al. Multidrug-resistant tuberculosis in Norway: a nationwide study, 19952014. Int J Tuberc Lung Dis 2016; 20: 786-792.

6 Zignol M, Dean AS, Alikhanova N, et al. Population-based resistance of Mycobacterium tuberculosis isolates to pyrazinamide and fluoroquinolones: results from a multicountry surveillance project. Lancet Infect Dis 2016; 16: 1185-1192.

7 Aung KJ, Van Deun A, Declercq E, et al. Successful '9-month Bangladesh regimen' for multidrug-resistant tuberculosis among over 500 consecutive patients. Int J Tuberc Lung Dis 2014; 18: 1180-1187.

8 Rigouts L, Coeck N, Gumusboga M, et al. Specific gyrA gene mutations predict poor treatment outcome in MDR-TB. J Antimicrob Chemother 2016; 71: 314-323.

9 Kuaban C, Noeske J, Rieder HL, et al. High effectiveness of a 12-month regimen for MDR-TB patients in Cameroon. Int J Tuberc Lung Dis 2015; 19: 517-524.

10 World Health Organization. Frequently asked questions about the implementation of the new WHO recommendation on the use of the shorter MDR-TB regimen under programmatic conditions. http://who.int/tb/ areas-of-work/drug-resistant-tb/treatment/FAQshorter_MDR_regimen.pdf. Date last updated: December 20, 2016. Date last accessed: January 25, 2017.

11 Fox W. Whither short-course chemotherapy? Br J Dis Chest 1981; 75: 331-357.

12 Fuentes ZM, Caminero JA. [Controversies in the treatment of extrapulmonary tuberculosis]. Arch Bronconeumol 2006; 42: 194-201.

13 Hoffmann H, Kohl TA, Hofmann-Thiel S, et al. Delamanid and bedaquiline resistance in Mycobacterium tuberculosis ancestral Beijing genotype causing extensively drug-resistant tuberculosis in a Tibetan refugee. Am J Respir Crit Care Med 2016; 193: 337-340.

14 Olaru ID, Lange C, Heyckendorf J. Personalized medicine for patients with MDR-TB. J Antimicrob Chemother 2016; 71: 852-855.

Copyright @ERS 2017

\section{From the authors:}

In response to our manuscript on the eligibility for shorter treatment of multidrug-resistant tuberculosis (MDR-TB) in the European Union (EU) [1], Heldal and co-workers highlight the limitations of the surveillance data that were used to estimate the proportion of MDR-TB cases eligible for the shorter regimen, and they question the criteria that we used to define eligibility.

The World Health Organization (WHO) criteria for eligibility state that patients exposed to second-line medicines included in the shorter MDR-TB regimen for $\geqslant 1$ month are not eligible. As information on exposure is not available in our surveillance data, we excluded all cases with previous treatment, realising that this was a conservative approach. The data from Latvia (V. Riekstina, Centre of Tuberculosis and Lung Diseases, Riga, Latvia; personal communication) provide an indication that our approach was indeed conservative. Taking this criticism into account, readers are able to ascertain from the data provided in figure 1 in our published study [1], that $524(16.9 \%)$ of the 3103 new cases were eligible for the shorter MDR-TB treatment, given the exclusion of extrapulmonary TB and those resistant to kanamycin/amikacin, moxifloxacin/ofloxacin or ethambutol.

Heldal and colleagues question our decision to consider ofloxacin-resistant cases as moxifloxacin-resistant. They argue that only $7 \%$ of ofloxacin-resistant strains have been found to be resistant to moxifloxacin. The data to which they refer are from Azerbaijan, Bangladesh, Belarus (Minsk city), Pakistan and South Africa (Gauteng and Kwazulu Natal). In our EU/European Economic Area (EEA) data, 816 rifampicin-resistant TB cases were tested for both ofloxacin and moxifloxacin. Out of 208 ofloxacin-resistant cases, 169 (81.2\%) were also resistant to moxifloxacin. Thus, in our setting, cross-resistance between ofloxacin and moxifloxacin is frequent, supporting our choice of using ofloxacin resistance as a proxy for moxifloxacin resistance to cover missing data (1768 out of 1774 cases were tested for ofloxacin and 386 out of 1774 for moxifloxacin).

$@$ ERSpublications

Strict application of criteria shows that $11 \%$ of MDR-TB cases are eligible for the shorter MDR-TB regimen in the EU http://ow.ly/p9aq30bt1AI

Cite this article as: van der Werf MJ, Ködmön C, Catchpole M. Shorter regimens for multidrug-resistant tuberculosis should also be applicable in Europe. Eur Respir J 2017; 49: 1700463 [https://doi.org/10.1183/ 13993003.00463-2017]. 
The authors also challenge the fact that we considered MDR-TB cases with resistance to ethambutol as ineligible for the shorter MDR-TB regimen. We based our exclusion on the WHO criteria that for patients "who have documented or are likely to have strains resistant to medicines in the [shorter MDR-TB] regimen" [2] the new regimen should not be used [3]. As ethambutol is part of the shorter regimen, cases with reported resistance to ethambutol were considered ineligible in our analysis. It is questioned by Heldal and co-workers whether ethambutol resistance should be an exclusion criterion since the test for ethambutol resistance is unreliable, according to the WHO. In the EU/EEA, the growth-based (liquid) drug-susceptibility test (DST) methods (e.g. the mycobacteria growth indicator tube system) are widely used, especially in high-income countries. These methods have been found to be reliable for most of the anti-TB drugs [4]. Discrepancies amongst DST results obtained using the growth-based tests have been reported for ethambutol, in comparison with proportion methods (e.g. agar proportion) and $e m b \mathrm{~B}$ mutation analysis. Proportion methods and $e m b \mathrm{~B}$ mutation analysis more frequently indicate ethambutol resistance compared with growth-based methods [5]. Thus, ethambutol resistance may be underdiagnosed in the EU/EEA due to the preferred use of growth-based methods. Therefore, we believe that we were conservative in considering ethambutol-resistant MDR-TB cases ineligible. If ethambutol resistance had not been an exclusion criterion, 602 of the 1774 MDR-TB cases who only had additional resistance to ethambutol would have been eligible for the shorter MDR-TB regimen.

The authors also remark on the reliability of pyrazinamide resistance. However, information on pyrazinamide resistance is not available in our database and was therefore not considered.

We agree with Heldal and colleagues that the shorter MDR-TB regimen is also likely to be effective in extrapulmonary TB cases. However, as discussed, we followed the WHO eligibility criteria and therefore excluded extrapulmonary TB cases. It is hoped that data will soon be available to support the use of the shorter regimen in extrapulmonary MDR-TB cases.

We applaud the fact that Norway included the shorter regimens in their MDR-TB treatment options and we would welcome data from operational research in a European setting on the effectiveness of the shorter regimen in patients who are currently excluded.

Several papers have recently been published on this topic [6-11]. This is an indication of the increased interest in shorter MDR-TB treatment regimens. It is hoped that this interest will result in further studies that might provide evidence for the revised criteria, meaning more MDR-TB patients will be eligible for the shorter regimen.

Marieke J. van der Werf, Csaba Ködmön and Mike Catchpole

European Centre for Disease Prevention and Control, Stockholm, Sweden.

Correspondence: Marieke J. van der Werf, European Centre for Disease Prevention and Control, Granits väg 8, 17165 Solna, Sweden. E-mail: Marieke.vanderWerf@ecdc.europa.eu

Received: March 062017 | Accepted after revision: March 092017

Conflict of interest: None declared.

\section{References}

1 van der Werf MJ, Hollo V, Ködmön C, et al. Eligibility for shorter treatment of multidrug-resistant tuberculosis in the European Union. Eur Respir J 2017; 49: 1601992.

2 World Health Organization. WHO treatment guidelines for drug-resistant tuberculosis - 2016 update. Geneva, World Health Organization, 2016.

3 Falzon D, Schünemann HJ, Harausz E, et al. World Health Organization treatment guidelines for drug-resistant tuberculosis, 2016 update. Eur Respir J 2017; 49: 1602308.

4 Yakrus MA, Driscoll J, McAlister A, et al. Molecular and growth-based drug susceptibility testing of Mycobacterium tuberculosis complex for ethambutol resistance in the United States. Tuberc Res Treat 2016; 2016: 3404860.

5 Rusch-Gerdes S, Domehl C, Nardi G, et al. Multicenter evaluation of the mycobacteria growth indicator tube for testing susceptibility of Mycobacterium tuberculosis to first-line drugs. J Clin Microbiol 1999; 37: 45-48.

6 Javaid A, Ahmad N, Khan AH, et al. Applicability of the World Health Organization recommended new shorter regimen in a multidrug-resistant tuberculosis high burden country. Eur Respir J 2017; 49: 1601967.

7 Lange C, Duarte R, Frechet-Jachym M, et al. Limited benefit of the new shorter multidrug-resistant tuberculosis regimen in Europe. Am J Respir Crit Care Med 2016; 194: 1029-1031.

8 Varaine F, Guglielmetti L, Huerga $\mathrm{H}$, et al. Eligibility for the shorter multidrug-resistant tuberculosis regimen: ambiguities in the World Health Organization recommendations. Am J Respir Crit Care Med 2016; 194: 1028-1029.

9 Sotgiu G, Tiberi S, D'Ambrosio L, et al. WHO recommendations on shorter treatment of multidrug-resistant tuberculosis. Lancet 2016; 387: 2486-2487.

10 Sotgiu G, Tiberi S, D’Ambrosio L, et al. Faster for less: the new "shorter" regimen for multidrug-resistant tuberculosis. Eur Respir J 2016; 48: 1503-1507.

11 Dalcolmo M, Gayoso R, Sotgiu G, et al. Resistance profile of drugs composing the "shorter" regimen for multidrug-resistant tuberculosis in Brazil, 2000-2015. Eur Respir J 2017; 49: 1602309. 